\title{
Adaptive Variability in Skilled Human Movements
}

\author{
Kazutoshi Kudo \\ Graduate School of Arts and Sciences, Department of Life Sciences, The University \\ of Tokyo \\ kudo@idaten.c.u-tokyo.ac.jp \\ Tatsuyuki Ohtsuki \\ (affiliation as previous author) \\ ohtsuki@idaten.c.u-tokyo.ac.jp
}

keywords: human, motor skill, motor control and learning, variability, brain, dynamical systems approach

\begin{abstract}
Summary
Human movements are produced in variable external/internal environments. Because of this variability, the same motor command can result in quite different movement patterns. Therefore, to produce skilled movements humans must coordinate the variability, not try to exclude it. In addition, because human movements are produced in redundant and complex systems, a combination of variability should be observed in different anatomical/physiological levels. In this paper, we introduce our research about human movement variability that shows remarkable coordination among components, and between organism and environment. We also introduce nonlinear dynamical models that can describe a variety of movements as a self-organization of a dynamical system, because the dynamical systems approach is a major candidate to understand the principle underlying organization of varying systems with huge degrees-of-freedom.
\end{abstract}

\section{Introduction}

Humans can perform a variety of skilled movements that any artificial machines or robots can not do. Since the precise mechanism of skilled motor control is still unknown, scientific exploration is needed to understand human motor skills, or the highest motor achievements of human beings.

To achieve highly skilled motor performance, a lot of problems occur due to variable nature of our external/internal environments. In fact, people frequently feel their movements fluctuate when they putt a golf ball, play the piano, or throw a basketball. Probably there is no one who does not have an experience of getting annoyed with such movement variability. Why do human movements vary?

One of the reasons is that humans have huge degrees of freedom in the motor system [Bernstein 67, Bernstein 96]. There are many joints that can be flexed/extended, abducted/adducted, or supinated/ pronated, and multiple muscles are often attached to a single joint. Each muscle has thousands of fibers to contract and exert force. The number of degrees of freedom increases as a viewpoint of analysis comes to more microscopic level in the physiological system.
Thus, when people perform a certain task using many joints, or even using a single joint, they have to deal with these huge numbers of degrees of freedom. It should be difficult and inefficient to decide how individual degrees of freedom should act at a time.

The individual degrees of freedom are subject to variation. Indeed many researchers have considered how a motor system does with its inherent variability. This problem is called a motor equivalence problem. Motor equivalence is defined as " the capacity of a motor system to achieve the same end-product with considerable variation in the individual components that contribute to that output" [Hughes 76]. Therefore, movement variability has been recognized as an important issue in the area of motor control. However, many researches have focused on the presumed noise or random sources of movement variability as discussed in the following section, ignoring adaptive aspects of variability.

\section{$1 \cdot 1$ Traditional View on Movement Variabil- ity: Variability as Noise}

Traditionally, movement variability has been discussed in relation to an error in motor performance 
[Schmidt 79, Welford 68]. In this view, variability has been considered as a troublesome source that should be eliminated from a motor system. As Schmidt et al. [Schmidt 79] put it:

The alternative we present in this article is based on the ideas that running a motor program results in muscle contractions (in turn, causing a particular pattern of movement), and that the mechanisms involved in this chain of events introduce noise (within-subject variability). The basis of the theory is an analysis of the role of the motor-output variability (noise) in producing movement inaccuracy and, more importantly, the role of certain movement variables (such as the movement distance, movement time, and mass to be moved) in determining the accuracy of the motor system. (p. 416)

Here, it is clearly stated that movement variability is equivalent to noise. Based on these traditional views, a lot of models have been proposed for human motor control. These models include deterministic iterative-corrections model [Keele 68], impulse variability model [Schmidt 79], and stochastic-optimized submovement model [Meyer 88]. In these models movement variability is produced by noise, which can be superimposed at a variety of levels in the deterministic relation between inputs and outputs.

In this sense, reducing variability has been considered as one of the most important factors in skill acquisition. Indeed, a lot of studies have suggested that both movement outcomes and movement patterns become more consistent with practice [Darling 87a, McDonald 89, Salthouse 86]. Moreover, there have been a lot of studies that observed highly consistent movement patterns in expert performers in ball games, such as baseball [Hore 96], table tennis [Tyldesley 75], and field hockey [Franks 85].

\subsection{Contribution}

In this paper, we introduce our research about human movement variability that shows remarkable coordination among components, and between organism and environment, that is, adaptive aspect of variability. In a series of research investigated motor tasks include a variety of movements such as ball-throwing, reaching, rhythmical tapping, speeded and precisely timed response, bipedal walking, swinging with pendulums, and playing musical instruments. In a theo- retical point of view, we introduce nonlinear dynamical models that can describe a variety of skilled motor control as a self-organization of a dynamical system, because the dynamical systems approach is a major candidate to understand the principle underlying organization of varying system with huge degrees-offreedom.

We believe that the viewpoint of adaptive variability in complicated and complex systems will contribute to future development of artificial intelligence research.

\section{Variability as a Result of Adjust- ment: Adaptive Variability}

An expert basketball player like Michael Jordan might be able to perform consistent free throws all the time. However, the movements are never exactly the same from trial to trial if precisely measured. That is, skilled movements can be highly consistent, but cannot be exactly the same. The question then arises about whether variability of consistent movements is produced merely by noise in a neuromuscular system or not.

Recent investigations have found evidences that there are other causes of variability; that is, variability as a result of adjustment, compensation, or coordination has been found by several experiments [Arutyunyan 69, Bootsma 90, Darling 87b, Vereijken 92]. These investigations strongly suggest that variable movement in which components are complementarily coordinated can produce consistent outcome. Therefore, we investigated this type of movement variability by developing a new index to measure the degree of compensation or coordination.

\subsection{Compensatory coordination among move- ment parameters}

How humans deal with the variability, when they are required to perform accurate movements? To answer this question, we examined organization of variable movement parameters in ball-throwing task aimed at a stationary target [Kudo 00].

It is reasonable that consistent movements produce consistent outcomes in a static environment. As for a throwing task aimed at a stationary target, if throwing movements become consistent, the release parameters (i.e., the release point, the magnitude and direction of release velocity vector), which inevitably determine the trajectory of the projectile, will become consistent (Figure 1A). As a consequence, the perfor- 
A

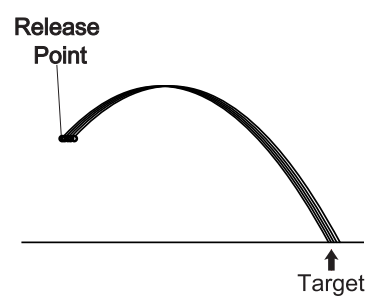

B

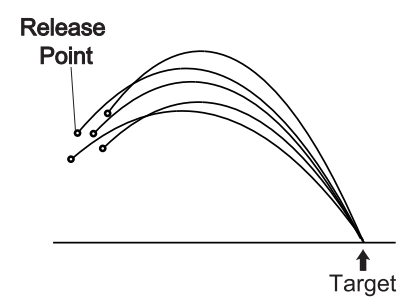

Fig. 1 Two possible solutions for accurate throw. A. Solution by consistent parameters. B. Solution by compensatory combination of variable parameters.

mance will become consistent. In a throwing task, consistency within the release parameters is a sufficient condition for performance consistency, but it is not a necessary condition because there are redundant degrees of freedom in determining the release parameters [Hamilton 97, Landa 79]. Thus, consistent throwing performance can be produced by variable release parameters coordinated in such a way that the trajectory of the projectile will reach the target (Figure 1B).

Therefore, we investigated whether or not there are compensatory relationships among the release parameters in a ball throwing task and whether or not this coordination improves during practice. In order to answer these questions we developed a new index called ICRP (index of coordination for release parameters).

The ICRP is an index to quantify the amount of increase in performance consistency in a given block of trials due to coordination among the release parameters, not due to consistency of the parameters. The ICRP is expressed as the ratio of two variability measures: variability of performance (standard deviation of the range of ball flight) calculated by all the possible combinations of the release parameters over variability of performance calculated by the original (i.e., actually observed) release parameters. If the release parameters are complementarily coordinated with one another, then variability of performance should increase after the coordinative relation is randomized or broken. On the other hand, if the release parameters do not compensate one another, then variability of performance will not increase after the randomization.

Concretely, the calculation of the ICRP includes the following steps. Suppose a subject stand just behind of a criterion position, or $(0,0)$ in Cartesian coordinates and throws a ball at a target placed on the floor

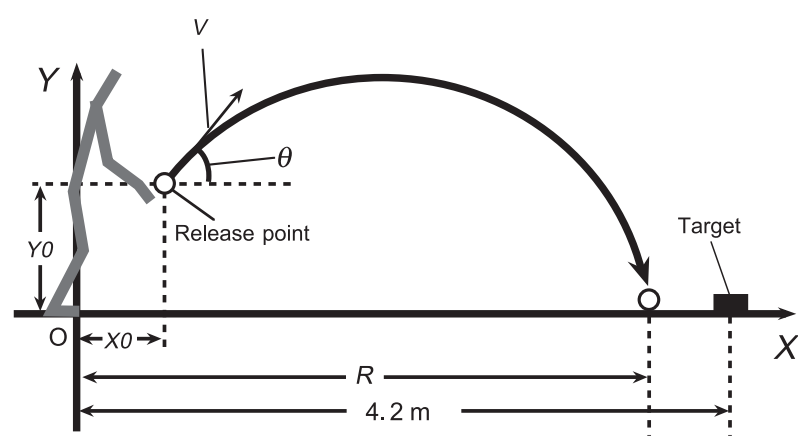

Fig. 2 The coordinate system to describe ball-relaese parameters and performace.

(Figure 2), then a set of release parameters, that is, the horizontal and vertical coordinates of the point of ball release (X0, Y0), the direction of ball-release velocity vector $(\theta)$, and the magnitude of ball-release velocity vector $(\mathrm{V})$ can be obtained from the coordinates.

The range of ball flight $(R)$ can be expressed as the following equation:

$$
\begin{aligned}
R= & \frac{V^{2} \cos \theta}{g}\left(\sin \theta+\sqrt{\sin ^{2} \theta+\frac{2 g Y 0}{V^{2}}}\right) \\
& +X 0 .
\end{aligned}
$$

Using Equation 1, we calculate the range for the $i$ th trial from the observed release parameters. Then the variability or standard deviation $(S D)$ of the ranges in a block of $n$ trials $\left(\sigma R_{\text {origial }}\right)$ is given by

$$
\sigma R_{\text {origial }}=\sqrt{\frac{1}{n} \sum_{i=1}^{n}\left(R_{i}-\bar{R}\right)^{2}},
$$

where $\bar{R}$ indicates the mean of $R_{i}$. The second step is calculation of variability from randomized release parameters among trials. Theoretically, unbiased estimate of $\sigma R$ calculated from randomized release parameters $\left(\sigma R_{\text {randomized }}\right)$ is obtained by,

$$
\sigma R_{\text {randomized }}=\sqrt{\frac{\zeta(n)}{\left(n^{3}\right)^{2}}-\frac{\eta(n)}{\left(n^{3}\right)^{2}}},
$$

where

$$
\begin{aligned}
& \zeta(n)=\sum_{i=1}^{n} \sum_{j=1}^{n} \sum_{k=1}^{n} \\
& \left\{\frac{V_{i}^{2} \cos \theta_{j}}{g}\left(\sin \theta_{j}+\sqrt{\sin ^{2} \theta_{j}+\frac{2 g Y 0_{k}}{V_{i}^{2}}}\right)+X 0_{k}\right\}
\end{aligned}
$$



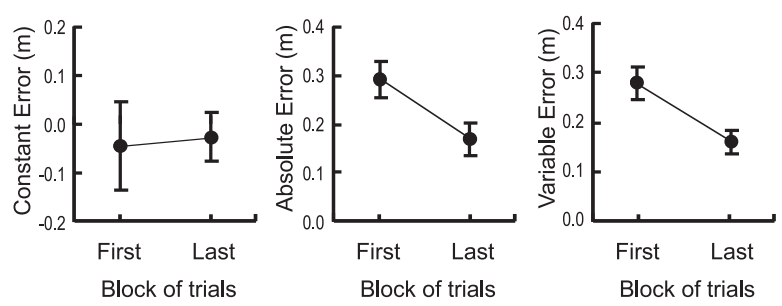

Fig. 3 Performce measures for the first and last block of trials. Each block consists of 30 trials.

and

$$
\begin{aligned}
& \eta(n)=\sum_{i=1}^{n} \sum_{j=1}^{n} \sum_{k=1}^{n} \\
& \left\{\frac{V_{i}^{2} \cos \theta_{j}}{g}\left(\sin \theta_{j}+\sqrt{\sin ^{2} \theta_{j}+\frac{2 g Y 0_{k}}{V_{i}^{2}}}\right)+X 0_{k}\right\}^{2}
\end{aligned}
$$

Finally, the ICRP is given as the ratio of $\left(\sigma R_{\text {randomized }}\right)$ over $\left(\sigma R_{\text {origial }}\right)$, that is,

$$
I C R P=\frac{\sigma R_{\text {randomized }}}{\sigma R_{\text {origial }}}
$$

This index represents the degree of compensatory coordination of the release parameters in a given block of trials. If the release parameters do not compensate for one another, then $\sigma R_{\text {origial }}$ should not be different from $\sigma R_{\text {randomized }}$. One the other hand, any compensatory relationships among the parameters should result in the greater $\sigma R_{\text {randomized }}$ than $\sigma R_{\text {origial. }}$. In the experiment, eight participants practiced ball-throwing task for five blocks of 30 trials, a total of 150 times.

Figure 3 illustrates constant error, absolute error, and variable error for each trial block (For calculation of these errors, please see Schmidt [Schmidt 88]). The results indicated that while constant error did not significantly change between the trial blocks $(t=-.58$, $d f=7, p>.58)$, both absolute and variable errors became smaller for the last block than for the first block $(t \mathrm{~s}>4.74, d f=7, p \mathrm{~s}<.002)$. These results indicated that while there were no significant differences in performance bias between the trial blocks, performance became more accurate and consistent with practice for 150 trials. The mean and within-participant $S D$ of the release parameters indicated that only the withinparticipant $S D$ of the initial speed $(V)$ significantly reduced with practice, while the other parameters

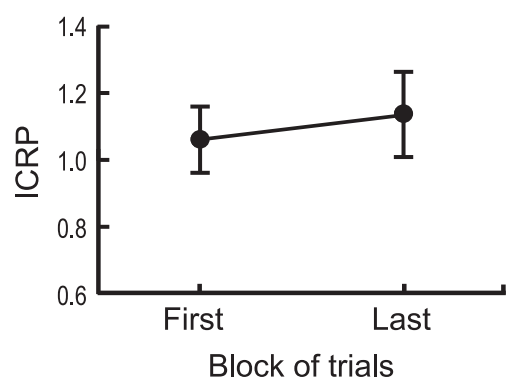

Fig. 4 Averaged index of coordination for release parameters (ICRP) for the first and last blocks of trials.

(i.e., $X, Y$, and initial angle) did not significantly change.

Figure 4 illustrates the ICRP score averaged across subjects for the first and last blocks. The averaged ICRP score was 1.06 for the first block and 1.12 for the last block. This means that variability of the performance would increase by $6 \%$ and $12 \%$ for the first and the last blocks, respectively, when the coordination of the parameters was randomized or broken.

The degree of coordination was relatively lower for the first block than the last block, suggesting the relationships among parameters were relatively noisy over successive trials as Mitra, Amazeen, and Turvey [Mitra 98] argued that noisiness is a characteristics of behavior during early phase of motor skill learning. On the other hand, the compensatory coordination among parameters tended to improve for the last block $(t=2.12, d f=7, p<.07)$. This suggests that variability in the release parameters in the last block was an emergence of active and functional compensatory process at least in part, and was not merely caused by noise or random process [Bootsma 90, Newell 93].

Such a compensatory relationship among multiple parameters as seen in this experiment can be explained by the concept of synergy, which is defined as taskdependent structural unit working toward a common goal [Bernstein 67, Gelfand 71]. Bernstein [Bernstein 67] argued that the synergy can solve the redundant degrees-of-freedom problem by introducing dependencies between components of motor system.

\subsection{Colored variability in simple movements}

Movements in the same external environment can be variable because of internal variability. If movement variability is a simple reflection of internal noise, one can observe the same type of noise throughout the movement. Therefore, we investigated the type 
of variability (color of noise) and possible transition of the type of variability during simple movements [Kadota 04, Miyazaki 01, Miyazaki 04].

Miyazaki et al. [Miyazaki 01] investigated the timedependent property of the kinematic variability in human rapid goal-directed movements by using fractal analysis. The variability of the peak acceleration had fractal time correlation (i.e., pink noise). The color became washy at the peak velocity and nearly bleached at the movement endpoint, indicating that during movement execution the variability became white noise with no time correlation.

These results suggest that background variability underlying motor process is pink noise, as is often found in many systems. The fractal correlation became relatively lower at the initial trajectory and vanished at the end, probably as a result of precise control. These observations are clearly against the notion that movement variability is produced by white noise that is superimposed in the stimulus input-response output processes.

\section{Electromyographic (EMG) Variabil- ity in Response to Changing Envi- ronment}

A tennis ball, which is hit by an opponent player, never draws the same trajectory during a game. Not only the direction of the ball but also the speed varies from trial to trial. Thus, a player has to deal with varying, or changing environments in a game. In addition, every time the player tries to stroke the ball, the player's posture is never the same. In these cases, the same movements produce variable and erroneous performance outcomes. Therefore, movements have to be variable, or in other word to be modified or adjusted every time, in order to obtain consistent performance. In particular, when the environment changes suddenly, the movement has to be modified rapidly.

A very rapid movement executed within $200 \mathrm{~ms}$ has been called“ ballistic" movement, as Rothwell [Rothwell 94] put it:

\section{Ballistic limb movements}

These are voluntary movements of a limb made as rapidly as possible from one target position to another. The movement is made quite freely without end stops in the apparatus, so that the subject has to reach and maintain the final position himself. The movements
A

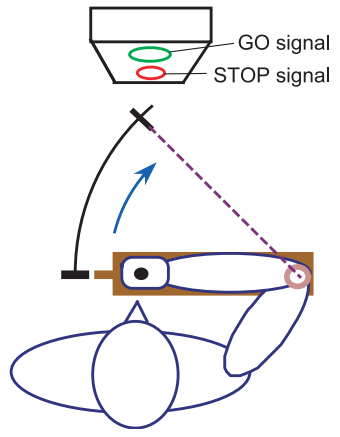

B

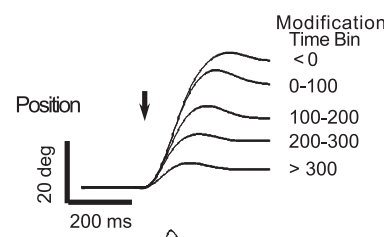

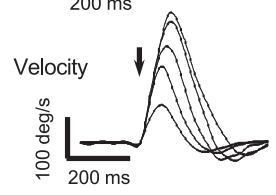

Fig. 5 Rapid movement modification task. A. Apparatus setting. B. Ensembled average of the position and velocity of movement in response to STOP signal. Vertical arrows indicate movement onset time.

are completed in $200 \mathrm{~ms}$ or less, which is so short that the subject is unable to make any voluntary corrections to the ongoing movement... The movements are made with a relatively stereotyped pattern of muscle activity. (pp. 433-434)

Schmidt [Schmidt 88] also claimed that these rapid movements are" preprogrammed, or structured in advance, and run off as a unit without much modification from events in the environment." Taken together, it has been considered that a rapid movement executed within $200 \mathrm{~ms}$ or so is difficult to be modified once it is initiated.

Therefore, we examined the possibility of rapid modification in response to sudden changes in the environment [Kudo 95, Kudo 98]. In the experiment, eight participants were required to rapidly extent the elbow joint in response to GO signal and to rapidly inhibit the ongoing movement in response to unexpected STOP signal that was presented after GO signal in $45 \%$ of trials (Figure $5 \mathrm{~A}$ ). The interstimulus interval (ISI), or time interval between the go and stop signals was randomly varied between 0 and 200 ms.

Subjects could sometimes completely inhibit initiation of the movements when the ISI was $0 \mathrm{~ms}$, but could rarely do so when the ISI exceeded $100 \mathrm{~ms}$. For responses that were initiated but stopped on the way, the peak amplitude of the movement linearly decreased as the time interval from the stop signal to the agonist EMG onset (=modification time) increased (Figure 5B). The peak velocity linearly increased as the movement amplitude increased. This tendency was similar to those previously reported in step-tracking movements with various amplitudes [Fre- 

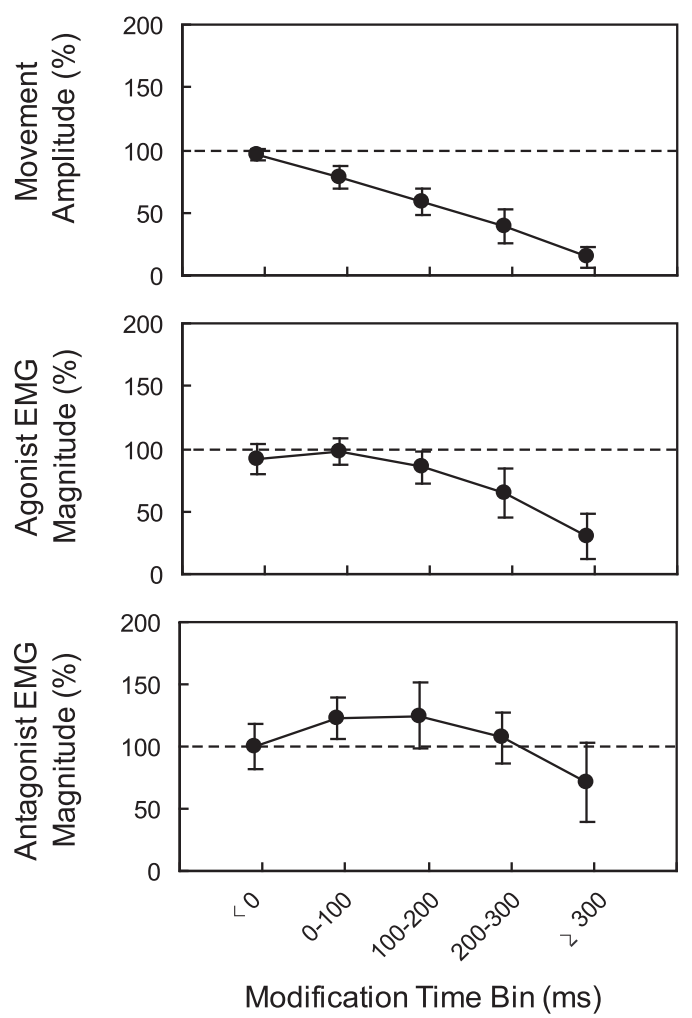

Fig. 6 Peak movement amplitude and EMG magnitude as a function of modification time. EMG magnitudes are expressed as percentage of EMG magnitude in the trials without STOP signal.

und 78]. In spite of the similarity in the kinematics of the movement, the EMG pattern was different from that of the general step-tracking movements (Figure 6 ). While the initial agonist burst linearly decreased after the modification time exceeded $100 \mathrm{~ms}$, the antagonist burst increased compared to go trial for the modification time from 0 to $200 \mathrm{~ms}$ and decreased after modification time exceeded $300 \mathrm{~ms}$.

These results suggest that even" ballistic" movements can be modified in response to the sudden changes in the environment. In the experiment, such a rapid modification was accomplished by adaptive, compensatory coordination of agonist-antagonist EMG activities. Therefore, we conclude that compensatory variability observed as a functional synergy also exists in muscle activity level.

\section{Adaptive Variability in Brain Activ- ity}

\section{4-1 Task-dependent activation and deactiva- tion of brain activity to identical stimu- lus}

Human brain consists of many interrelated structures such as cerebellar cortex, cerebellum, basal ganglia, and so on. Such brain modules are required
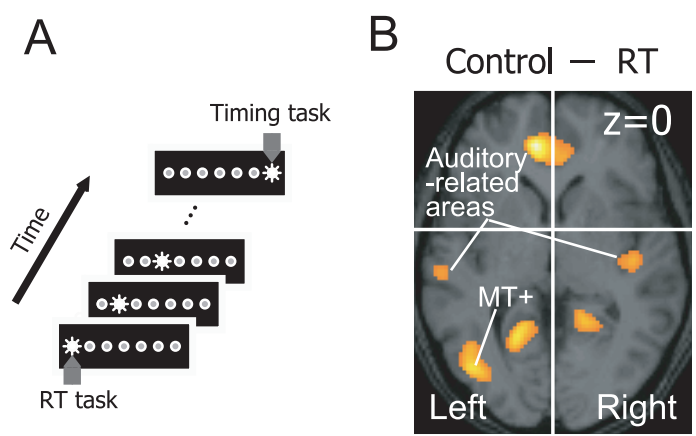

Fig. 7 Context-dependent brain activity in response to identical stilmuli. A. Stimulus used. Individual lights were presented one after another from side to side. B. Deactivated brain area in reaction time (RT) task. The brain image is shown in the transaxial plane at $z=0$ in the Tarailach coordinates [Talairach 88]. Horizontal and vertical lines indicate $x=0$ and $y=0$, respectively.

to be active or inactive depending on the task demands. Therefore, the authors [Kudo 04] investigated task-dependent modification of brain activity in response to the identical stimulus using fMRI (functional magnetic resonance imaging). In this experiment 12 participants were asked to execute right index finger/thumb tapping using the right hand in response to the first stimulus in the reaction time (RT) task and in anticipation of the last stimulus in the timing task (Figure 7A). In control condition the same participants were asked to passively view the stimulus without motor response.

Results showed that various movement-related areas including contralateral cingulate motor cortex were commonly activated for both tasks relative to the control condition, suggesting these structures are involved in general perception and response execution rather than specific function for speeded or precisely timed responses. In the RT task the pre-supplementary motor area extending to the cingulate sulcus was activated more strongly than in the timing task probably to focus attention to the onset of the first stimulus unpredictably presented after random foreperiods. The lateral occipital area extending to the temporoparieto-occipital junction (MT+ area) was activated more strongly in the timing task than in the RT task, and the same area was deactivated in the RT task relative to the control condition (Figure 7B). Auditoryrelated areas were also deactivated in the both tasks.

These findings underscore importance of attention and intention for perception and action, because high sensitivity to some characteristics of stimulus (e.g., motion) in a certain brain area does not necessarily 
mean that the area is automatically activated for the stimulus. We demonstrated that the area that is potentially be activated by a certain stimulus can be deactivated for the same stimulus depending on the context. These findings also underscore importance of selective activation/deactivation in brain activity due to selective attention or top-down attentional control [Hopfinger 00] for skilled performance, because usually in natural environment there are full of information that can potentially interfere with one another and should be selectively perceived for relevant motor execution.

\subsection{Functionally equivalent motor task is re- alized by different combination of brain activation}

The environment surrounding us is abundant. This means that different types of information can specify the same event. For example, a certain time in the future can be specified by two consecutive onsets of stimuli as well as by time-to-arrival information [Lee 81]. Therefore, using fMRI, the authors [Kudo 01] investigated human brain activity for interval and interceptive timing tasks, both of which require functionally equivalent timing response. Participants were asked to tap their thumb with the index finger to indicate the arrival time of a moving stimulus at a target location in the interceptive task, and to anticipate the time of occurrence of consecutive stimuli in the interval timing task. The results demonstrated that functionally equivalent timing response can be executed by using different types of information and different brain structures.

Results showed that the human basal ganglia, that is hypothesized as an internal timekeeper in temporal processing [Rao 01], was activated only in the interval timing task condition. Therefore, functionally equivalent response such as the time production in the present experiment could be executed by using different types of information and different brain structures. This underscores the importance of utilization of information not only in the skilled movement but also movements by the patients with disabilities in the brain because patients with a particular brain damage can perform functionally equivalent responses if they are provided appropriate information for the remaining intact brain structures.

\section{Dynamical Systems Approach: Pos- sible Theoretical Background under Adaptive Variability}

Dynamical system approach, that has successfully explained a variety of complex phenomena as a selforganization of a nonequilibrium open system, is a major candidate to understand the principle underlying organization of huge degrees-of-freedom system at various levels including movement, EMG activity, and brain activity level [Haken 78, Haken 96, Kelso 95, Kugler 87, Taga 91]. Practical implication of the dynamical systems approach for physical education or coaching was also discussed by Yamamoto [Yamamoto 02].

\section{$5 \cdot 1$ Nonlinear dynamical system can overcome the variability problem}

The authors conducted theoretical study on nonlinear dynamical system and demonstrated that the dynamical system can overcome the problem of internal variability [Ohgane 04]. In human physiological system, variable time delay can destabilize smooth motor execution. For example, variable delay in the sensorimotor loop can cause fall in walking. Ohgane et al. [Ohgane 04] demonstrated that stable bipedal locomotion under variable sensorimotor delay can be attained by a simulation model with nonlinear oscillators that produce flexible-phase locking. In their model, central pattern generator (CPG) was modeled by the following differential equations,

$$
\left\{\begin{aligned}
& \tau_{i} \dot{u}_{i}(t)= u_{i}(t)-v_{i}(t)-u_{i}(t)^{3} / 3 \\
&+\sum_{i j=1}^{12} w_{i j} y_{i}+u_{0}+\alpha F_{i}\left(\mathbf{x}\left(t-\Delta_{a}\right)\right) \\
& \tau_{i}^{\prime} \dot{v}_{i}(t)=u_{i}(t)+a-b v_{i}(t) \\
& y_{i}=f\left(u_{i}(t)\right) \\
& f(u)=\max (0, u)
\end{aligned}\right.
$$

where $u_{i}$ is the potential of the $i$ th neuron, $v_{i}$ is responsible for the accommodation and refractoriness of the $i$ th neuron, $w_{i j}$ is the connecting weight from the $i$ th neuron to the $j$ th neuron, $\tau_{i}$ and $\tau_{i}^{\prime}$ are the time constants of the inner state and the accommodation and refractory effects, respectively, $y_{i}$ is the output of the $i$ th neuron, $u_{0}$ is the constant parameter, $\alpha$ is a coupling strength from the body to the CPG, $F_{i}$ is a sensory feedback, $\mathbf{x}$ is a $(6 \times 1)$ vector of the mass 
point positions of 1 link and the inertial angles of 4 links, $t$ is the time, and $a$ and $b$ are positive constants.

The simulation showed that locomotion control adaptive to time delay could emerge from the delayed coupling of two dynamics formed by the CPG and the body. Ohgane et al [Ohgane 05] also demonstrated that flexible walking model can overcome more severe internal variability such as sudden ankle joint impairment as a result of an injury.

\subsection{Environmental coupling}

Human movements consist of huge redundant degrees of freedom at various anatomical and physiological levels. Bernstein [Bernstein 67] proposed that individual degrees of freedom are organized into larger functional unit called synergy. It is important to recognize that these synergies should be organized not only among subsystems but also between a system and environment because various task constraints are imposed by the environment. In such a case, environmental coupling becomes an important factor in system's realizing coordinative flexibility and multistability in continuously changing environment. Rhythmic bimanual coordination can be modeled by a motion equation in the collective variable of relative phase $\phi=\left(\theta_{L}-\theta_{R}\right)$, the difference in the phase angles $(\theta)$ of the left and right limbs. A solution of the motion equation

$$
\dot{\phi}=-a \sin \phi-2 b \sin 2 \phi+\sqrt{Q} \xi_{t}
$$

is $\phi$ 's stable state $\phi *$ for the current parameterization of the coordination [Haken 85, Schöner 86]. The overdot in Equation 8 signifies $\phi$ 's rate of change. The parameters of the sine functions in $\phi$ and $2 \phi$ in Equation 8 determine the relative strengths of the stable or attractive states at or near $\phi=0^{\circ}$ (in-phase coordination) and $\phi=180^{\circ}$ (anti-phase coordination). The final right-hand term in Equation 8 is a Gaussian white noise process $\xi_{t}$ of strength $Q>0$. Theory and research identify changes of $a$ and $b$ with the coupled movement frequency. If $b / a>.25$, the fixed points or attractors of Equation 8 are $\phi=0^{\circ}$ and $\phi=180^{\circ}$, respectively. Although both attractors constrain coordination within this parameter range, Equation 8 indictates that they do not do so with equal force. Anti-phase is less strongly attractive than in-phase. The contrast is revealed by $\lambda$, the value of $d \dot{\phi} / d \phi$ evaluated at $\phi *$. $\lambda$ is the Lyapunov exponent for the region near $\phi *$. The“ strength" or" attractiveness" of $\phi *$ is determined by the magnitude of $|\lambda|$ where $1 / \lambda$ is a time - the time to return or relax to the attractor following a brief perturbation. This magnitude is less for $\phi *=180^{\circ}$ (anti-phase) than $\phi *=0^{\circ}$ (inphase). For both attractors, $|\lambda|$ decreases as $b / a$ decreases. Formally, $S D \phi *$ is determined by $Q$ and $\lambda$ [Gilmore 81]:

$$
S D \phi^{*}=\sqrt{\frac{Q}{2|\lambda|}}
$$

Theoretically, the stability of bimanual coordination is characterized by the following quantities: attractor location $\phi *$, system noise $Q$ in the attractor state, and attractor strength $\lambda$. We can ask therefore, how does the strength of environmental coupling affect $\phi *, Q$, and $\lambda$. To answer this question, Kudo et al. [Kudo 06] analyzed the time series of pendulum oscillation by cross recurrence quantification (CRQ). In CRQ, time delayed copies of the respective time series are used as surrogate dimensions required for embedding the data in higher dimensional space to examine recurrent structure between paired signals [Zbilut 98]. The method can detect subtle nonlinear dynamics of weakly coupled oscillators. The CRQ revealed that environmental coupling that was controlled through metronome beat modified system's stability in terms of noise strength and noise sensitivity.

Two CRQ measures are of special significance to bimanual rhythmic coordination. One measure, \%RECUR, is the number of points that are recurrent, expressed as a proportion of the total number of points. It reflects the locations shared by the left and right oscillations in phase space. It is the proportion of all possible points where the trajectories of the two rhythmic processes cross. The other measure, MAXLINE, is the longest continuous sequence of points in phase space expressed as a proportion of the longest sequence possible. This measure reflects the stability of shared activity (convergence of nearby trajectories over time). Formally, system noise $Q$ and attractor strength $\lambda$ can be estimated with \%RECUR and MAXLINE measurements calculated by CRQ method, respectively.

In the experiment, nine participants performed inphase pendulum oscillations as smoothly and accurately as possible in synchronization to the auditory metronome. Environmental coupling was controlled though metronome beat (one beat or two beats per cycle). The metronome, set to emit one or two beeps 
A

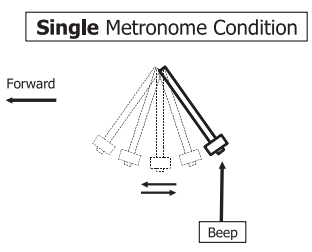

Double Metronome Condition
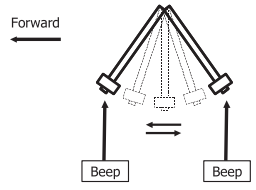

B
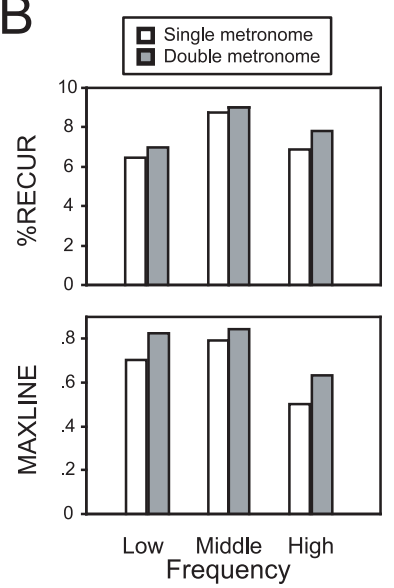

Fig. 8 Environmental coupling experiment. A. Single and double metronome condition. B. \%RECUR and MAXLINE for each metronome and frequency condition.

per cycle, marked the frequency of movement at 0.67 , 1.00, or $1.33 \mathrm{~Hz}$. Participants were instructed to synchronize with the metronome at the most forward position of the in-phase pendulum motions when there was a single beep and at both the extreme forward and backward positions when there were two metronome beeps (Figure 8A).

We estimated $Q$ and $\lambda$ using CRQ (Figure 8B). Results indicated that environmental coupling reduces the amount of system noise and increases attractor strength. From these findings, the environmental coupling concept may extend to coordination more generally. Functional groupings of muscles (synergies or coordinative structures) have demonstrated flexibility in a variety of tasks including speech [Kelso 84], ballthrowing [Hirashima 03a, Hirashima 03b, Kudo 00], rapid movement modification [Kudo 98], and bipedal locomotion [Ohgane 04]. On the present findings these functional, flexible organizations can incorporate environmental components to enhance adaptability and stability. The findings encourage inquiry into " embedded coordination" : the study of coordination as the dynamics of a human-environment system and not simply of the human.

\subsection{Source of movement variability in expert drummers and non-drummers}

Highly skilled performances result from long-term practice. For example, expert musicians who have practiced intensively for a long time demonstrate highly stable and impressive performances. In our recent studies [Fujii ed, Kudo 07], we successfully described professional drummers' and non-drummers' bimanual

performance by the following differential equation;

$$
\begin{aligned}
\dot{\phi}= & -a \sin \phi-2 b \sin 2 \phi-c \sin (\phi-\psi) \\
& +\Delta \omega+d \Delta \omega \cos \phi+\sqrt{Q} \xi_{t}
\end{aligned}
$$

In Equation 10, $\phi$ denote the relative phase between the left and right hand movement. The $a$ and $b$ parameters determine the relative strengths of the attractive states near $\phi=0^{\circ}$ and $\phi=180^{\circ}$. The third term, $c \sin (\phi-\psi)$ represents intentional dynamics [Kelso 95]. The term corresponds to an intentional force or effort to stabilize the intended relative phase pattern $\left(\psi=180^{\circ}\right)$ of a given strength $c$. When the intentional force to stabilize the antiphase pattern can override the inherent dynamic tendencies of the motor system (i.e., $\psi=180^{\circ}$ and $c>>a, b$ ), the system has only one stable pattern, reflecting the ability of participants to maintain the antiphase pattern, even at a high movement frequency. Phase wandering observed in non-drummers' performance (Figure 9) was modeled by introducing a symmetry breaking term $\Delta \omega$. As $\Delta \omega$ increases, the stable fixed point disappears, causing the phase wandering to appear. In addition, as $\Delta \omega$ increases, the mean also increases. To keep the mean $\phi$ at $180^{\circ}$, another intentional term is needed. We introduced the term as $\Delta \omega \cos \phi$. The final righthand term is a Gaussian white noise process $\xi_{t}$ of strength $Q>0$.

We simulated the relative phase pattern of drummers and non-drummers performing alternate (i.e., antiphase) stroke by using Equation 9. In the simulation, we changed only the $\Delta \omega$ parameter $(\Delta \omega$ values are 0.62 and $1.61 \mathrm{rad} / \mathrm{s}$ for drummers and nondrummers, respectively), fixing the other parameters at $a=$ $1, b=0, c=2, d=0.8$, and $\sqrt{Q}=20$. As seen in Figure 9 , the simulated results successfully reproduced the observed pattern, suggesting that $\Delta \omega$ is a critical learning parameter, constraining the performance of bimanual drumming. It should be noted that symmetry breaking parmeter $\Delta \omega$ rather than noise strength $Q$ is critical in determining performance stability and variability. Previous studies have assumed that $\Delta \omega$ represents a difference in eigenfrequency between two components [Kelso 95, Kugler 87]. Our findings revealed that $\Delta \omega$ can denote a functional asymmetry of the upper limbs, or neural constraints, because the left and right limbs are anatomically and mechanically equivalent.

In our model, a gradual decrease in $\Delta \omega$, or the intrinsic functional asymmetry of the upper limbs, pro- 


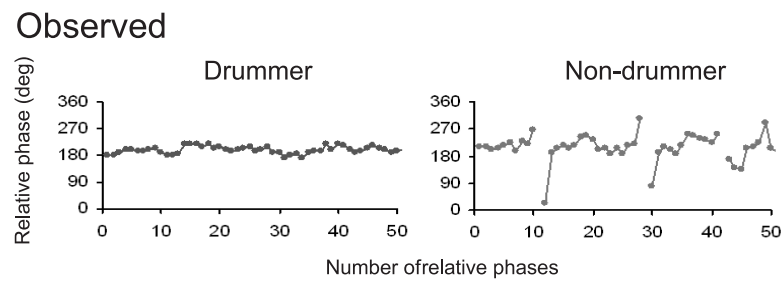

Simulated

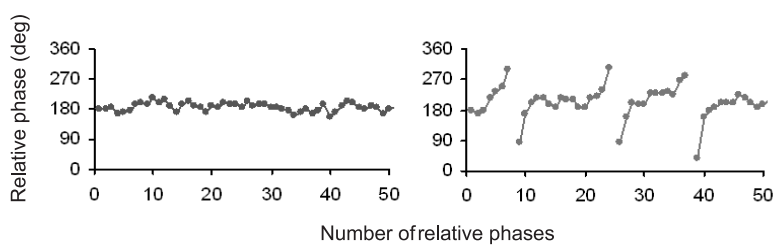

Fig. 9 Observed and simulated time series of the relative phase of basic drumming performance.

duces a drastic change in bimanual coordination patterns (i.e., from phase wandering mode to no phase wandering mode), and at the same time causes a spontaneous decrease in intentional effort for the required movements. This could allow expert musicians to instead devote effort to artistic expression, such as style, phrasing, and ensemble. By changing a control parameter that constrains performance, our model successfully described the process of automatization in motor learning. This dynamical system approach will enable us to concisely describe general expertization as an emergent property of a self-organized system.

\section{Conclusion}

As seen in the series of research, movements vary even if one tries to move consistently in a static environment. One of the sources of this variability is variability of our internal environment. For example, an activation level in $\alpha$ motoneuron pool, which influences recruitment of motor unit, is varying time after time. The amount of neurotransmitter in the motor end plate, which influences muscle contraction, is also never constant. Many other sources (e.g., the amount of ATP in muscle or membrane potential in afferent and efferent nerve) that have a potential to fluctuate movement are also continuously changing. Therefore, the same nerve impulse will end up with different muscle contraction and consequently with different movements. Hence it is very difficult, or rather impossible to reproduce constant movement even if we try to do so. In this sense, highly reproducible movement is not a result of recall or reproduction of a set of command stored as a memory but a consequence of continuous adjustment [Bernstein 67, Bernstein 96].

As far as movements vary due to variability in physiological states, adjustment of this variability is clearly needed to obtain consistent performance outcome. In addition, not only internal environment but also external environment is consistent when one tries to perform a motor skill. In these environments, compensatory coordination among parameters rather than fixation of the parameters is necessary to produce skilled movements. A large amount of evidence have suggested that skilled movements are produced by combining, coordinating, and coupling variabilities that exist in multiple levels of the internal and external environments. Thus, these variabilities should not be random variability produced merely by noise, but compensatory and adaptive variability. That is, adaptive variability actually plays a critical role in human motor control. We argue that such variability is a necessary condition to make a system be flexible and intelligent.

We also showed that dynamical systems approach can be a hopeful candidate to explain the organization of redundant and fluctuating degrees of freedom. It can describe human motor control and learning as time evolution or self-organization of a dynamical system.

In conclusion, not only human movements but also flexible intelligent systems will be, can be, and should be variable to achieve highly skilled motor performance.

\section{Acknowledgments}

This study was partly supported by the Japan Society for the Promotion of Science (JSPS) Grants-inAid for Scientific Research (No. 17500416) awarded to K.K.

\section{$\diamond$ References $\diamond$}

[Arutyunyan 69] Arutyunyan, G. A., Gurfinkel, V. S., and Mirskii, M. L.: Organization of movements on execution by man of an exact postural task, Biophysics, Vol. 14, pp. 1162-1167 (1969)

[Bernstein 67] Bernstein, N. A.: The coordination and regulation of movements, Pergamon Press, New York (1967)

[Bernstein 96] Bernstein, N. A.: On dexterity and its development, in Latash, M. L. and Turvey, M. T. eds., Dexterity and its development, pp. 1-244, Lawrence Erlbaum, New Jersey (1996)

[Bootsma 90] Bootsma, R. J. and Wieringen, van P. C. W.: Timing an attacking forehand drive in table tennis, Journal of Experimental Psychology: Human Perception and Performance, Vol. 16, pp. 21-29 (1990) 
[Darling 87a] Darling, W. G. and Cooke, J. D.: Changes in the variability of movement trajectories with practice, Journal of Motor Behavior, Vol. 19, pp. 291-309 (1987)

[Darling 87b] Darling, W. G. and Cooke, J. D.: Movement related EMGs become more variable during learning of fast, accurate movements, Journal of Motor Behavior, Vol. 19, pp. 311-331 (1987)

[Franks 85] Franks, I. M., Weicker, D., and Robertson, D. G. E.: The kinematics, movement phasing and timing of a skilled action in response to varying conditions of uncertainty, Human Movement Science, Vol. 4, pp. 91-105 (1985)

[Freund 78] Freund, H. J. and Budingen, H. J.: The relationship between speed and amplitude of the fastest voluntary contractions of human arm muscles, Experimental Brain Research, Vol. 31, pp. 1-12 (1978)

[Fujii ed] Fujii, S., Kudo, K., Ohtsuki, T., and Oda, S.: Expert musicians have acquired freedom from intrinsic constraint of asymmetry (submitted)

[Gelfand 71] Gelfand, I. M., Gurfinkel, V. S., Tsetlin, M. L., and Shik, M. L.: Some problems in the analysis of movements, in Gelfand, I. M., Gurfinkel, V. S., Fomin, S. V., and Tsetlin, M. L. eds., Models of the structural-functional organization of certain biological systems, pp. 329-345, MIT Press, London (1971)

[Gilmore 81] Gilmore, R.: Catastrophy theory for scientists and engineers, Wiley, NY (1981)

[Haken 78] Haken, H.: Synergetics: an introduction, Splinger Verlag, Berlin (1978)

[Haken 85] Haken, H., Kelso, J. A., and Bunz, H.: A theoretical model of phase transitions in human hand movements, Biological Cybernetics, Vol. 51, No. 5, pp. 347-56 (1985)

[Haken 96] Haken, H.: Principles of brain function, Springer Verlag, Berlin (1996)

[Hamilton 97] Hamilton, G. R. and Reinschmidt, C.: Optimal trajectory for the basketball free throw, Journal of Sports Sciences, Vol. 15, pp. 491-504 (1997)

[Hirashima 03a] Hirashima, M., Kudo, K., and Ohtsuki, T.: Utilization and compensation of interaction torques during ball-throwing movements, Journal of Neurophysiology, Vol. 89, pp. 1784-1796 (2003)

[Hirashima 03b] Hirashima, M., Ohgane, K., Kudo, K., Hase, K., and Ohtsuki, T.: Counteractive relationship between the interaction torque and muscle torque at the wrist is predestined in ball-throwing, Journal of Neurophysiology, Vol. 90, pp. $1449-1463$ (2003)

[Hopfinger 00] Hopfinger, J. B., Buonocore, M. H., and Mangun, G. R.: The neural mechanisms of top-down attentional control, Nature Neuroscience, Vol. 3, No. 3, pp. 284-91 (2000)

[Hore 96] Hore, J., Watts, S., and Tweed, D.: Errors in the control of joint rotations associated with inaccuracies in overarm throws, Journal of Neurophysiology, Vol. 75, No. 3, pp. 1013-1025 (1996)

[Hughes 76] Hughes, O. M. and Abbs, J. H.: Labialmandibular coordination in the production of speech: Implications for the operation of motor equivalence, Phonetica, Vol. 44, pp. 199-221 (1976)

[Kadota 04] Kadota, H., Kudo, K., and Ohtsuki, T.: Timeseries pattern changes related to movement rate in synchronized human tapping, Neuroscience Letters, Vol. 370, No. 2-3, pp. 97-101 (2004)

[Keele 68] Keele, S. W.: Movement control in skilled motor performance, Psychological Bulletin, Vol. 70, pp. 387-403 (1968)

[Kelso 84] Kelso, J. A. S., Tuller, B., Bateson, V. E., and Fowler, C. A.: Functionally specific articulatory cooperation following jaw perturbations during speech: Evidence for coordinative structures, Journal of Experimental Psychology: Human Perception and Performance, Vol. 10, No. 6, pp. 812-832 (1984)
[Kelso 95] Kelso, J. A. S.: Dynamic patterns, MIT Press, Cambridge (1995)

[Kudo 95] Kudo, K. and Inomata, K.: Ballistic movement can be anticipatively controlled, in Fu, F. H. and Ng, M.-. L. eds., Sport psychology: Perspectives and practices toward the 21st century, pp. 257-260, Glory, Hong Kong (1995)

[Kudo 98] Kudo, K. and Ohtsuki, T.: Functional modification of agonist-antagonist electromyographic activity for rapid movement inhibition, Experimental Brain Research, Vol. 122, pp. 23-30 (1998)

[Kudo 00] Kudo, K., Ito, T., Tsutsui, S., Yamamoto, Y., and Ishikura, T.: Compensatory coordination of release parameters in a throwing task, Journal of Motor Behavior, Vol. 32, No. 4, pp. 337-345 (2000)

[Kudo 01] Kudo, K., Miyazaki, M., Kimura, T., Yamanaka, K., Kadota, H., Hirashima, M., Nakajima, Y., Nakazawa, K., and Ohtsuki, T.: Differential brain activation in interval and interceptive timing tasks: an fMRI study, Neuroscience Research, Supplement, Vol. 25, p. 152 (2001)

[Kudo 04] Kudo, K., Miyazaki, M., Kimura, T., Yamanaka, K., Kadota, H., Hirashima, M., Nakajima, Y., Nakazawa, K., and Ohtsuki, T.: Selective activation and deactivation of the human brain structures between speeded and precisely timed tapping responses to identical visual stimulus: an fMRI study, NeuroImage, Vol. 22, No. 3, pp. 1291-301 (2004)

[Kudo 06] Kudo, K., Park, H., Kay, B. A., and Turvey, M. T.: Environmental coupling modulates the attractors of rhythmic coordination, Journal of Experimental Psychology: $\mathrm{Hu}$ man Perception and Performance, Vol. 32, No. 3, pp. 599609 (2006)

[Kudo 07] Kudo, K., Fujii, S., Ohtsuki, T., and Oda, S.: Motor Automaticity as an Emergent Property of a Dynamical System, in Cummins-Sebree, S., Riley, M. A., and Shockley, K. eds., Studies in Perception 83 Action IX, pp. 28-31, Lawrence Erlbaum, NJ (2007)

[Kugler 87] Kugler, P. N. and Turvey, M. T.: Information, natural law, and the self-assembly of rhythmic movement, Erlbaum, NJ, Hillsdale (1987)

[Landa 79] Landa, J.: Analysis of skill acquisition on a novel throwing task in terms of biomechanical factors, Journal of Human Movement Studies, Vol. 5, pp. 52-60 (1979)

[Lee 81] Lee, D. N. and Reddish, P. E.: Plummeting gannets: a paradigm of ecological optics, Nature, Vol. 293, pp. 293$294(1981)$

[McDonald 89] McDonald, P. V., Emmerik, van R. E. A. and Newell, K. M.: The effects of practice on limb kinematics in a throwing task, Journal of Motor Behavior, Vol. 21, pp. 245-264. (1989)

[Meyer 88] Meyer, D. E., Abrams, R. A., Kornblum, S., Wright, C. E., and Smith, J. E. K.: Optimality in human motor performance: Ideal control of rapid aimed movements, Psychological Review, Vol. 95, pp. 340-370 (1988)

[Mitra 98] Mitra, S., Amazeen, P. G., and Turvey, M. T.: Intermediate motor learning as decreasing active (dynamical) degrees of freedom, Human Movement Science, Vol. 17, pp. 17-65 (1998)

[Miyazaki 01] Miyazaki, M., Kadota, H., Kudo, K., Masani, K., and Ohtsuki, T.: Fractal correlation of initial trajectory dynamics vanishes at the movement end point in human rapid goal-directed movements, Neuroscience Letters, Vol. 304, pp. 173-176 (2001)

[Miyazaki 04] Miyazaki, M., Nakajima, Y., Kadota, H., Chitose, K., Ohtsuki, T., and Kudo, K.: 1/f-type fluctuation in human visuomotor transformation, Neuroreport, Vol. 15, No. 7, pp. 1133-1136 (2004)

[Newell 93] Newell, K. M. and Corcos, D. M.: Issues in variability and motor control, in Newell, K. M. and Corcos, D. M. eds., Variability and Motor Control, Human Kinetics, Champaign, IL (1993)

[Ohgane 04] Ohgane, K., Ei, S., Kudo, K., and Ohtsuki, T.: 
Emergence of adaptability to time delay in bipedal locomotion, Biological Cybernetics, Vol. 90, pp. 125-32 (2004)

[Ohgane 05] Ohgane, A., Ohgane, K., Ei, S., Mahara, H., and Ohtsuki, T.: Initial-state coordinations reproduce the instant flexibility for human walking, Biological Cybernetics, Vol. 93, pp. 426-435 (2005)

[Rothwell 94] Rothwell, J.: Control of Human Voluntary Movement, Chapman \& Hall, New York (1994)

[Salthouse 86] Salthouse, T. A.: Effects of practice on a typing-like keying task, Acta Psychologica, Vol. 62, pp. 189198 (1986)

[Schmidt 79] Schmidt, R. A., Zelaznik, H., Hawkins, B., Frank, J. S., and Quinn, J. T.: Motor Output Variability: Theory for the Accuracy of Rapid Motor Acts, Psychological Review, Vol. 86, No. 5, pp. 415-451 (1979)

[Schmidt 88] Schmidt, R. A.: Motor Control and Learning, Human Kinetics, Illinois (1988)

[Schöner 86] Schöner, G., Haken, H., and Kelso, J. A.: A stochastic theory of phase transitions in human hand movement, Biological Cybernetics, Vol. 53, No. 4, pp. 247-57 (1986)

[Taga 91] Taga, G., Yamaguchi, Y., and Shimizu, H.: Selforganized control of bipedal locomotion by neural oscillators in unpredictable environment, Biological Cybernetics, Vol. 65, No. 3, pp. 147-59 (1991)

[Talairach 88] Talairach, J. and Tournoux, P.: Co-planar stereotaxic atlas of the human brain, Georg Theime Verlag, New York (1988)

[Tyldesley 75] Tyldesley, D. and Whiting, H. T. A.: Operational timing, Journal of Human Movement Studies, Vol. 1, pp. 172-177 (1975)

[Vereijken 92] Vereijken, B., Vanemmerik, R. E. A., Whiting, H. T. A., and Newell, K. M.: Free(z)Ing Degrees of Freedom in Skill Acquisition, Journal of Motor Behavior, Vol. 24, No. 1, pp. 133-142 (1992)

[Welford 68] Welford, A. T.: Fundamentals of skill, Methuen, London (1968)

[Yamamoto 02] Yamamoto, Y.: Perspectives on motor learning: application of a dynamical systems approach, Japanese Journal of Physical Education, Health and Sport Sciences, Vol. 47, pp. 125-40 (2002)

[Zbilut 98] Zbilut, J. P., Giuliani, A., and Webber, J., C. L.: Detecting deterministic signals in exceptionally noisy environments using cross-recurrence quantification, Physics Letters A, Vol. 246, pp. 122-128 (1998)

\section{〔担当委員：藤波 努〕}

Received January 10, 2008.

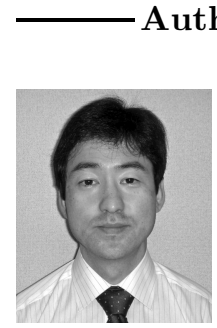

\section{Author's Profile}

\section{Kudo, Kazutoshi}

Kazutoshi Kudo received his Ph.D. in Life Sciences from the University of Tokyo in 1998. He was a visiting researcher at the Center for the Ecological Study of Perception and Action in the University of Connecticut from 2002 to 2003 . He is currently an assistant professor in the Graduate School of Arts and Sciences, the University of Tokyo. His research interests include skilled human motor control and dexterity. $\mathrm{He}$ is a member of Japan Society of Sport Psychology, Japanese Society for Ecological Psychology, Japan Neuroscience Society, Japan Society of Physical Education, Health and Sport Sciences, and Japan Society of Human Growth and Development.

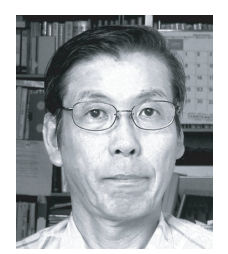

\section{Ohstuki, Tatsuyuki}

Tatsuyuki Ohtsuki received his Ph.D. in Education from the University of Tokyo. He was a Lecturer and an Associate Professor at Nara Women's University from 1975-1994. He is currently a Professor in the Graduate School of Arts and Sciences, the University of Tokyo. His areas of interest include neuro-psycho-biomechanical mechanisms of skilled human motor control. He is a member of the Japan Society of Physical Education, Health and Sport Sciences, the Japanese Society of Physical Fitness and Medicine, Japan Society of Biomechanics, Japan Society of Exercise and Sports Physiology, Japan Neuroscience Society, Japanese Society of Clinical Neurophysiology, Physiological Society of Japan, Human Ergology Society, Japan Society of Sport Psychology, American College of Sports Medicine, International Society of Biomechanics, and North American Society for Neuroscience. 